\title{
Clinical teaching strategies that inspire and get the most out of your learners
}

\section{By Allan Lai}

\section{Introduction}

$\mathrm{C}$

linical teaching has become a fundamental skill in emergency nursing. The demand for 24-hour emergency care has never been greater, and coupled with the global nursing shortage, emergency departments (ED) are recruiting and training more specialized emergency nurses to meet the demands. Technology is evolving at a rate that has never been witnessed in history, which, in turn, has led to innovations in patient treatment that greatly influence how ED nurses practise. Some argue that it is a professional responsibility for current emergency nurses to train and support the success of the next generation of emergency nurses.

The pressures facing EDs have been well documented. A lack of physical space to provide care, high acuity, and a seemingly revolving door of patients are all problems that nurses face when they teach. Complicating this are the ambiguous roles for the emergency nurse working with a learner: Should they be teaching or facilitating? How should they teach? Moreover, the pressures of time can give the impression that a deep exploration of clinical discussions should take a backseat to patient care.

Whereas the literature surrounding clinical teaching in emergency medicine is extensive, searches for literature on clinical teaching specific to emergency nursing yield limited results. To address this gap, this article will offer effective teaching strategies for emergency nurses hoping to inspire and get the most out of learners in the ED.

\section{A structured approach}

Studies exploring the characteristics of effective clinical instructors (as defined by students) have suggested that effective instructors promote autonomy and have expertise in the relevant clinical setting, strong feedback, and clear communication skills (Kelly, 2007; Mailloux, 2006; Tang, Chou, \& Chiang, 2005). To apply these characteristics to the ED, the following strategies are presented in an ABCDEFGHI mnemonic familiar to emergency nurses.

\section{Assess the learner, Build trust, Cue them, and Diagnose their learning}

Assessing and acknowledging the learner's experience is critical in establishing a clear and respectful relationship. Given that nearly all medical, surgical, and psychiatric conditions in varying degrees of acuity present to the ED, an understanding of the learner's prior experience will provide the means to identify the learner's stage of competence and transition (Kramer, 1974; Benner, 1984). For example, an emergency nurse trainee may have a wealth of experience in critical care and, thus, be comfortable with rapid sequence intubation. However, the trainee may not have been exposed to or have treated an undifferentiated pelvic bleed before. Cuing the learner to identify their learning needs and strategies based on their experience will empower them to decide how and what to learn. Putting the learner in the driver's seat facilitates their accountability for their own learning, which is in keeping with the principles of adult learning theory (Kaufman, 2002).

Having set the stage earlier allows the emergency nurse to diagnose the learner; that is, to identify what type of competence and transition stage they are experiencing. A learner identified as being keenly and consciously aware of their incompetence and who may be in transition shock requires mentorship, positive feedback, and normalization. On the other hand, a learner diagnosed as being unconsciously incompetent and in a honeymoon phase would require realistic expectation setting (Kramer, 1974).

\section{Expectation setting, Facilitation, Giving feedback with clean language}

The emergency nurse and the learner should set expectations at the beginning of clinical, and they should mutually evaluate their progress routinely throughout clinical training. Expectations should be set in relation to the learner's competence. The majority of the time, emergency nursing students are novices in their approach to ED patients. It's therefore necessary to set realistic expectations to reflect their novice level. For example, an emergency nursing student may have the goal of planning and delivering care to a patient suffering from anaphylaxis, but their speed and skill may not be fully developed. In this example, the emergency nurse should share their concern for acuity beyond the learner's skill level, and they should come to a mutual agreement with the learner regarding the best way to approach the patient.

When applying the principles of adult learning theory to our learners, emergency nurses must recognize that trainees are autonomous and problem-focused, that they learn from their mistakes, and that true theory conceptualization must be relevant to the practice (Kaufman 2002). This method of delivering education is less about "teaching" and more about facilitation. For example, allowing the learner to see a new patient arriving to a care space independently is much more beneficial for their learning than "tag-teaming" a patient without the learner's consent. As the facilitator, the emergency nurse should ensure that key patient treatment priorities are not delayed, such as timely electrocardiograms for acute chest pain.

ED patients often have incomplete histories or no collateral information. Allowing the learner to experience this independently, sort through their line of questioning, and make minor mistakes will let the learner truly grasp the practice of emergency nursing. Once the learner has reached a conclusion or stalled in their progress, the emergency nurse can facilitate the learner's 
experience by asking clear, concise questions that may have more than one acceptable answer. These questions are generally open-ended and genuine, and they facilitate learner reflection. One such example would be: "What made you choose to apply continuous SpO2 monitoring?” Avoid asking leading questions where a specific answer is wanted, such as: "What three conditions require SpO2 monitoring?”

Using clean language, such as debriefing with good judgment, is a skill that can further enhance learner reflection. Advocacy/ inquiry is a method of clean questioning where the emergency nurse reveals their frame of reference while genuinely inquiring about the reason for the learner's action or inaction (Rudolph et al, 2007). For example, the emergency nurse may choose to ask a question using advocacy/inquiry such as: "I noticed you did not apply an $\mathrm{SpO} 2$ probe in your primary survey. I felt it would've helped your decision-making. Help me understand your thought process." This approach allows the emergency nurse to reveal their perspective and prevents the learner from guessing what their teacher wants them to say while simultaneously allowing for a genuine answer. The learner may respond with an answer that can generate rich clinical discussion, such as: "I did not think the SpO2 reading would have changed my management because the fingers were cold and we would treat the dyspnea regardless of the $\mathrm{SpO} 2$ reading." One powerful question can generate more learning points than multiple closed questions.

Effective constructive feedback in the emergency department must be respectful, concise, timely, and given only with the learner's permission. Mistakes will happen, and if used as learning opportunities, they serve as powerful teaching tools. While setting the stage of clinical, an emergency nurse who informs the learner that feedback will be shared gains their permission. This allows the relationship to be respectful, and trust is reinforced. Feedback should be objective, concise, timely, and devoid of jargon. The speed of the ED can cause the learner to forget and, in some cases, the learner may genuinely be unaware of their mistakes. Self-concept and motivation to learn are driving factors for learner success, and an emergency nurse can promote this by sharing positive feedback often. Both positive and constructive feedback should allow the learner to share their perspective.

\section{Hear and listen more/talk less \& Independence}

It has been found that teachers wait on average less than one second for a response to a question from a learner (Peninciner, 2002). The emergency nurse should be prepared for silence when waiting for an answer to a question. Be prepared to wait ten seconds or longer. This allows the learner to hear, deconstruct, interpret, synthesize, and formulate a response. In the event that patient care is a priority resulting in time limitations, inviting the learner to reach a conclusion and return when ready to share can be highly effective. Promoting this independence will motivate the learner, tap into their experience, and orient them to do more and memorize less.

\section{Conclusion}

Clinical teaching in busy emergency departments is both challenging and rewarding. Embracing the uniqueness of the ED and incorporating these qualities into teaching strategies can lead to rich learning experiences for emergency nursing students. Although these strategies are provided in a linear format here, like emergency assessment frameworks, one can be flexible and creative in implementing them.

\section{About the author}

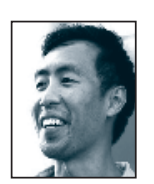

Allan Lai, BSN, RN, ENC(C), completed his BSN at Thompson Rivers University in Kamloops, BC. He currently practises as an emergency nurse at Vancouver General Hospital and teaches the emergency nursing specialty program at the British Columbia Institute of

Technology. He also supports the EPICC National Design Team in various ways, most recently as a project team member for the upcoming EPICC-Pediatrics course. 